\title{
Predictive factors for relapse of cryptogenic organizing pneumonia
}

\author{
Zenya Saito ${ }^{1 *} \mathbb{D}$, Yugo Kaneko', Tsukasa Hasegawa ${ }^{2}$, Masahiro Yoshida², Kyuto Odashima', Tsugumi Horikiri ${ }^{1}$, \\ Akira Kinoshita ${ }^{1}$, Keisuke Saitoh ${ }^{1}$ and Kazuyoshi Kuwano ${ }^{3}$
}

\begin{abstract}
Background: Relapse of cryptogenic organizing pneumonia (COP) may lead to poor long-term prognosis and necessitates multiple rounds of steroid treatment with potential adverse effects. The objective of this study is to identify predictive factors of COP relapse by comparing demographic and clinical variables between relapse and non-relapse groups.

Methods: During 2008-2013, 33 COP patients were treated, of which 23 (69.7\%) and 10 patients (30.3\%) were assigned to the non-relapse and relapse group, respectively. From medical records, we compared the following variables at initial episode: clinical characteristics, serum parameters, chest $C T$ scan findings, and steroid treatment.

Results: Clinical characteristics, cumulative prednisone dose, and steroid treatment duration were similar between groups. In univariate analysis, alternatively, the proportion of patients with bilateral shadow pattern, traction bronchiectasis, and partial remission after steroid treatment was significantly higher in the relapse group. These differences were not significant by multivariate Cox regression analysis.

Conclusions: We identified radiographic findings, such as bilateral shadow pattern, traction bronchiectasis, and partial remission, may have possibility of predictive factors for COP relapse. Larger-scale studies are required to confirm if any are independent predictors of COP relapse.
\end{abstract}

Keywords: COP, Predictive factor, Relapse, Steroid, Radiographic findings

\section{Background}

Organizing pneumonia $(\mathrm{OP})$ is pathologically defined by the presence of granulation tissue buds within distal pulmonary airspaces consisting of fibroblasts and myofibroblasts intermixed with loose connective matrix [1]. Clinically, OP is associated with a syndrome of acute or subacute onset that may include cough, fever, and progressive dyspnea, and radiographically, with multiple patchy alveolar opacities not responsive to antibiotics.

Organizing pneumonia is classified as either cryptogenic OP (COP), which has no specific etiology, or secondary OP caused by an inflammatory reaction to drugs, infection, collagen vascular disease, malignancy, or radiation therapy [2-4]. One previous study [5] reported that secondary OP was less responsive to treatment and

\footnotetext{
* Correspondence: zyst_0404@yahoo.co.jp

${ }^{1}$ Division of Respiratory Diseases, Department of Internal Medicine, The Jikei University Daisan Hospital, 4-11-1 Izumihoncho, Komae-shi, Tokyo 201-8601, Japan

Full list of author information is available at the end of the article
}

associated with worse prognosis than COP, while another [6] reported similar clinical and radiographic findings, treatment responses, relapse rates, and mortality for COP and secondary OP. Patients with OP sometimes relapse during or after steroid treatment, and several relapses increase the side effects of steroid treatment and may negatively influence prognosis. The frequency of relapse ranges from 9 to 33\% [7-9], and we sometimes encounter relapse when steroids are tapered or stopped. Therefore, it is important to identify predictive factors of relapse as possible aids to guide preventative treatment.

A relationship between relapse and steroid dose was identified [10, 11], and guidelines for steroid treatment have been proposed by Epler [3] and Schwartz and King [4]. Epler suggested starting with $1 \mathrm{mg} / \mathrm{kg} /$ day prednisone (60 mg/day) for $1-3$ months, tapered to $40 \mathrm{mg} /$ day for 3 months, and then 10-20 mg/day for 1 year. Schwartz and King suggested initiating therapy with $1-1.5 \mathrm{mg} / \mathrm{kg} /$ day prednisone for $4-8$ weeks, tapering to $0.5-1 \mathrm{mg} / \mathrm{kg} /$ day 
for the ensuing 4-6 weeks. Although there is general consensus on steroid efficacy, the specifics of the steroid treatment regimen, such as initial dose and interval of tapering, have not been standardized. Several studies reported that relapse was associated with tapering or discontinue of steroid treatment $[6,12]$. Other proposed predictive factors for relapse are severity of initial hypoxemia, traction bronchiectasis and architectural distortion on computed tomography (CT) scans with increased serum Krebs von den Lungen-6 (KL-6) levels, the presence of intra-alveolar fibrin in lung biopsy tissue, and the delay between first symptom and treatment onset [10, 12-15]. However, the predictive factors for relapse remain uncertain.

The purpose of this study is to identify predictive factors for relapse of COP by comparing the clinical characteristics, laboratory data, radiographic findings, duration of steroid treatment, cumulative equivalent dose of prednisone, and response to steroid therapy between non-relapse and relapse groups.

\section{Methods}

\section{Study population and selection criteria}

We retrospectively reviewed the medical record of patients admitted to the Jikei University Daisan Hospital between April 2008 and March 2013. The following criteria were required for inclusion in the present study: (1) histopathological reports based on transbronchial lung biopsy expressly describing the presence of granulation tissue buds in the distal airspaces, (2) clinical and imaging features compatible with COP such as fever, cough, dyspnea, and good response to steroid treatment, and (3) steroid treatment for COP. Patients were excluded for the following criteria: (1) secondary OP induced by infections, drugs, or collagen vascular diseases, (2) spontaneous improvement without treatment, or (3) malignant tumors. Patients were classified as relapse or non-relapse based on standard criteria (below). This study was approved by the institutional research ethics committee of the Jikei University School of Medicine, and written informed consent was obtained from all patients. All patient records were anonymized prior to analysis.

\section{Definition of relapse}

Relapse was defined as follows: (1) the appearance of characteristic new infiltrates and/or the exacerbation of residual target shadow on chest $\mathrm{CT}$ scans as well as COP-compatible clinical features during or after steroid treatment, (2) the improvement by increasing steroid treatment only without antibiotics. Cases conforming to this definition did not require histopathologic proof of COP for the diagnosis of relapse.
Typical images of non-relapse and relapse case were presented in Figs. 1 and 2.

\section{Data analysis}

We retrospectively compared the following variables from medical records at initial episode between relapse and non-relapse patients: sex, age, smoking status, symptoms and signs, white blood cell (WBC) count, lactate dehydrogenase (LDH), alkaline phosphatase (ALP), $C$ reactive protein $(C R P), K L-6$, surfactant protein $D$ (SP-D), chest CT scan findings (pattern and location of lesion), steroid treatment duration, cumulative equivalent dose of prednisone $(\mathrm{mg} / \mathrm{kg})$, reaction to steroid therapy. In reaction to steroid therapy, we compared the proportion of partial remission between groups and defined it as the presence of residual shadow that had not completely disappeared during tapering or after the completion of steroid treatment in CT scans.

\section{Review of radiology}

All patients had chest CT scans. Interpretation reports of all CT scans by radiologists were available for review. The pattern of abnormal findings was classified as consolidation, ground-glass attenuation, or nodules. The images were assessed for the distribution of lung parenchymal findings and the presence of sequelae such as traction bronchiectasis and pleural effusion. The distribution of abnormal findings was classified as unilateral or bilateral and involving the upper, middle, or lower lung zones. Regarding the distribution, the type of shadow wasn't distinguished. Ultimately all chest CT scans were reviewed and interpreted by two radiologists and three respiratory physicians, and discussions were held on findings if necessary. Finally, findings were identified by agreement of members.

The definitions of radiological terms [16] are as follows: consolidation was defined as areas of increased attenuation obscuring normal lung markings, ground-glass opacity as hazy attenuation without obscuration of the underlying vessels, nodules as coin-like shadows $<3 \mathrm{~cm}$ in diameter, and traction bronchiectasis as irregular bronchial dilatation surrounding parenchymal abnormalities caused by repeated cycles of infection and inflammation.

\section{Review of shadow distribution}

We reviewed the shadow distribution from CT scans to investigate the relationship between relapse and shadow distribution. The right lung has three lobes; upper, middle and lower. The left lung has two lobes: upper and lower. Each lobe is supplied by an individual lobar bronchus. Right upper lobe contains 3 segments (apical, posterior, and anterior), right middle lobe 2 (lateral and medial) and right lower lobe 5 (superior, medial, anterior, 


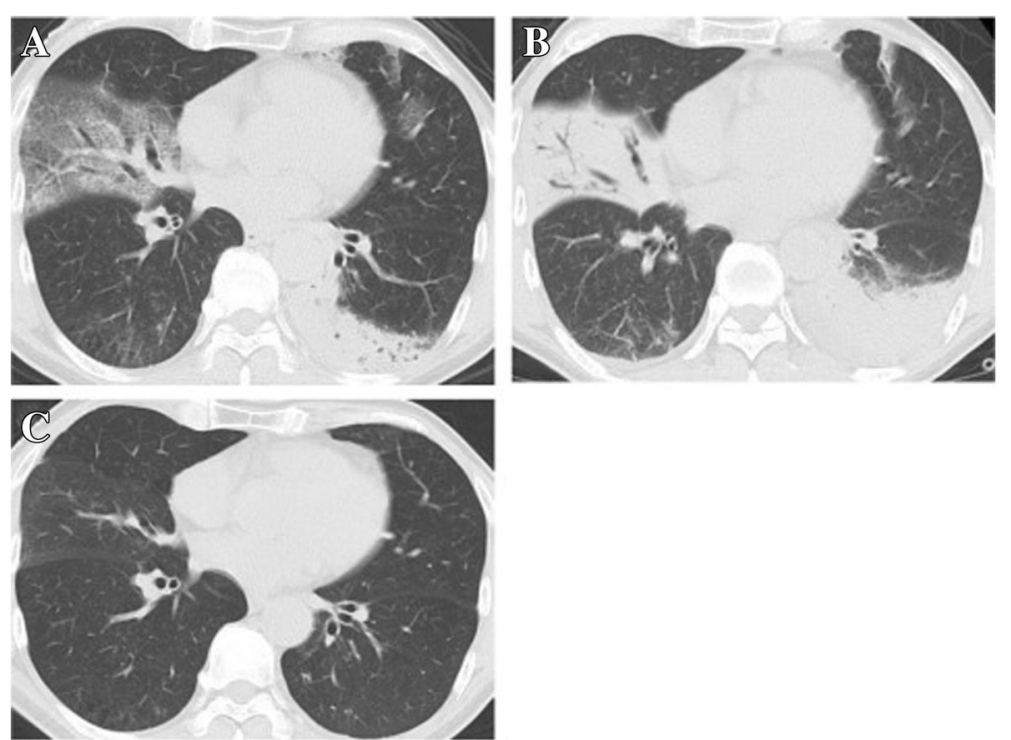

Fig. 1 Typical CT images of non-relapse case. a Ground-glass attenuation with traction bronchiectasis in the right middle lobe and consolidation in the left lower lobe were recognized at the first visit. $\mathbf{b}$ Ground-glass attenuation changed into consolidation in the right middle lobe and consolidation apparently expanded in the left lower lobe just before treatment. c All shadows completely vanished after three months and relapse didn't occur after one year

lateral, and posterior). Left upper lobe contains 4 segments (apicoposterior, anterior, superior lingular, and inferior lingular) and left lower lobe 3 (superior, anteromedial, lateral, and posterior). Based on these anatomical structures, we distributed all shadows on $\mathrm{CT}$ images to each lobe.

\section{Review of treatment}

We retrospectively examined the treatment records of all patients. To evaluate the relationship between relapse and steroid treatment, we expressed steroid dose in prednisone equivalents and compared the cumulative dose (in $\mathrm{mg} / \mathrm{kg}$ ) between relapse and non-relapse groups. Additionally, we compared the differences in total steroid treatment period and follow-up period between these groups.

\section{Statistical analysis}

The Mann-Whitney U test was used to compare means of continuous variables and the chi-square test to
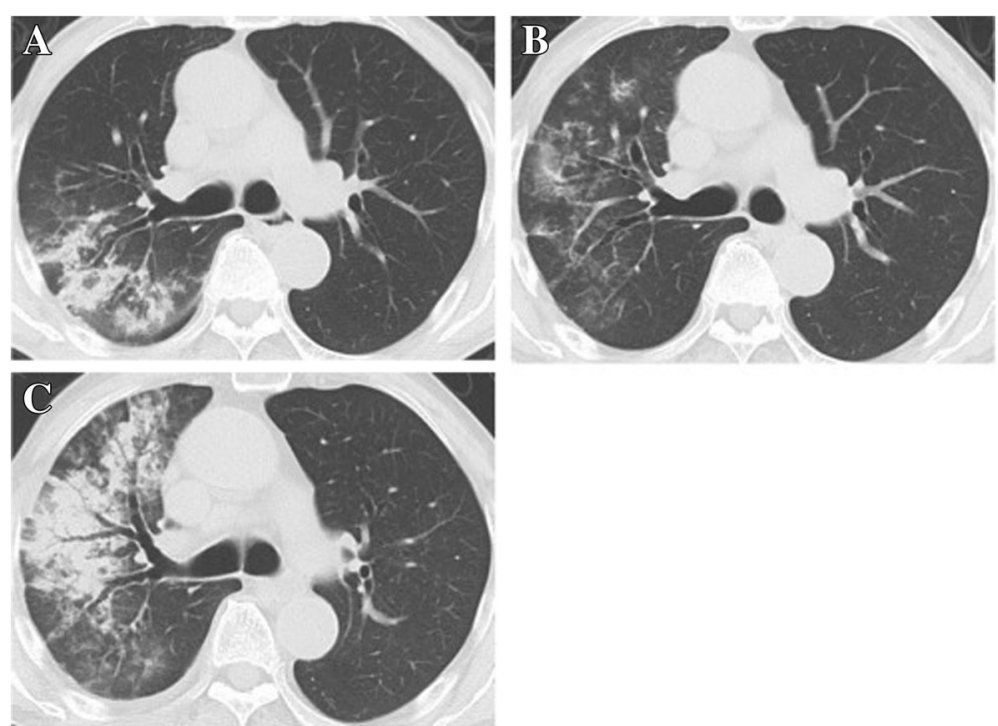

Fig. 2 Typical CT images of relapse case. a Consolidation and nodules in the right upper lobe were recognized at the first visit. $\mathbf{b}$ These shadows remained without vanishing completely after treatment. c Relapse occurred after six months of treatment 
compare proportions between groups. Multivariate Cox regression analysis was performed to evaluate the associations of clinical, and radiological features with relapse. A $P$ value $<0.05$ indicated statistical significance for all analyses. All statistical analyses were performed with Statistical Package for the Social Sciences software, version 20.0 (SPSS Inc., Chicago, IL, USA).

\section{Results}

Thirty-three patients fulfilled the selection criteria, of whom 10 (30.3\%) relapsed and 23 (69.7\%) did not (Fig. 3). The clinical characteristics of both groups are shown in Table 1, and there was no significant difference. The most common symptom in all patients was cough $(81.8 \%)$, followed by fever $(66.6 \%)$. One patient in the non-relapse group presented with chest pain. Physical findings included crackles in $60.6 \%$ and wheezing in $15.1 \%$ of all patients.

Four patients were still on steroid treatment (mean prednisone dose $6.8 \pm 3.7 \mathrm{mg}$; range $2.5-12.5 \mathrm{mg} /$ day) when the first relapse occurred. The mean time to first relapse was $476 \pm 445$ days (range, $70-1682$ days). All patients were treated with increased steroid dose when relapse occurred, and the mean prednisone equivalent dose at that time was $24 \pm 8 \mathrm{mg} /$ day.

Comparisons of serum parameters (Table 2) revealed no significant differences between groups. Table 3 compared the radiological findings between groups. Chest radiographs revealed consolidation in 30 patients (90.9\%), which was bilateral in 20 (60.6\%). The proportion of patients with a bilateral shadow pattern was significantly higher in the relapse group ( $90 \%$ vs. only $47.8 \%$ in the non-relapse group, $p=0.02$ ). Similarly, incidence of traction bronchiectasis was also significantly higher in the relapse group $(60 \%$ vs. $17.3 \%$ in the non-relapse group, $\mathrm{p}=0.02$ ). Although there was no significant difference in the lesion pattern distribution between groups (consolidation, ground-glass attenuation, or nodules), the proportion of patients with partial remission after steroid treatment was significantly higher in the relapse group ( $90 \%$ vs. $43.3 \%$ in the non-relapse group, $p=0.01$ ). Migratory infiltrative shadows were observed in five patients in the entire cohort (15.1\%) and did not differ significantly between groups.

There was no significant difference in the mean period of steroid treatment or cumulative equivalent dose of prednisone between groups (Table 4). All patients underwent antibiotic therapy before starting the steroid treatment. Eleven patients with severe respiratory disorder at onset received high-dose pulse intravenous steroid treatment with $1 \mathrm{~g}$ methylprednisolone for 3 days (30.4\% in the non-relapse group vs. $40 \%$ in the relapse group, NS).

Thus, the relapse group included a significantly higher proportion of patients with bilateral shadow pattern, traction bronchiectasis, and partial remission as indicated by univariate analysis. However, there were no significant differences by multivariate Cox regression analysis.

\section{Discussion}

This result that radiographic findings may be more important factor than clinical findings, and therapeutic passage is worth investigating further in the large-scale trials. As a result, we identified the three radiographic features, such as bilateral shadow pattern, traction bronchiectasis, and signs of partial remission (residual shadow), may have possibility the predictive factors of relapse. Four of the 10 patients (40\%) in the relapse group exhibited all these three radiographic factors, far higher than that in the non-relapse group $(8.7 \%)$, and three of the four patients had multiple relapses. Okada

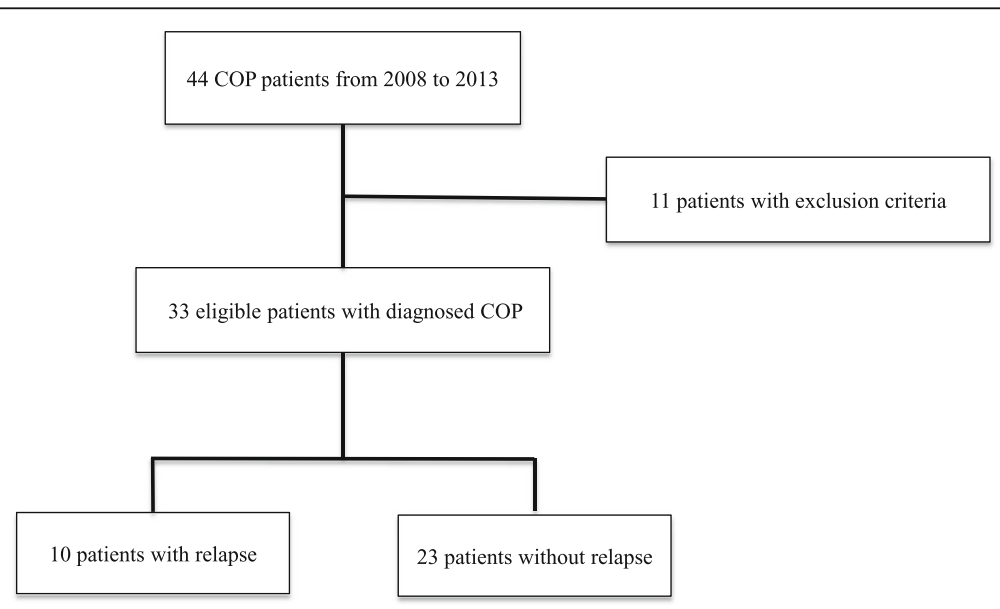

Fig. 3 Patient selection flow chart. Of the 33 eligible patients diagnosed as COP, 10 patients were classified to relapse group, and 23 patients were non-relapse 
Table 1 Comparison of clinical characteristics between non-relapse and relapse groups at initial episode in univariate analysis

\begin{tabular}{llll}
\hline Variable & $\begin{array}{l}\text { Non-relapse } \\
\text { group }(n=23)\end{array}$ & $\begin{array}{l}\text { Relapse group } \\
(n=10)\end{array}$ & P \\
\hline Age \pm SD & $74 \pm 14$ & $71 \pm 10$ & NS \\
$\geq 70$ years & $8(35)$ & $2(20)$ & NS \\
Male & $14(61)$ & $4(40)$ & NS \\
Female & $9(39)$ & $6(60)$ & NS \\
Smoking history & & & \\
$\quad$ Never smoked & $12(52)$ & $8(80)$ & NS \\
$\quad$ Prior smoker & $8(35)$ & $2(20)$ & NS \\
$\quad$ Current smoker & $3(13)$ & $1(10)$ & NS \\
Cough & $19(83)$ & $8(80)$ & NS \\
Sputum & $13(57)$ & $1(10)$ & NS \\
Dyspnea & $14(61)$ & $6(60)$ & NS \\
Fever & $15(65)$ & $7(70)$ & NS \\
Chest pain & $1(4)$ & $0(0)$ & NS \\
Crackles & $15(65)$ & $5(50)$ & NS \\
Wheezing & $4(17)$ & $1(10)$ &
\end{tabular}

Values are presented as No. (\%). Abbreviation: NS not significant

et al. [14] reported that CT findings of traction bronchiectasis and architectural distortion in COP patients were associated with serum KL-6 levels. In the present study, however, there was no significant difference in serum KL- 6 between groups, but 3 of the 10 relapse patients had both serum KL- $6>500 \mathrm{U} / \mathrm{ml}$ and traction bronchiectasis on chest images.

As regards steroid treatment of COP, there was no significant difference in the mean period of steroid treatment or cumulative equivalent dose between groups. There was a possibility that some patients with COP might have unnecessary and excessive steroid treatment. We need to comprehensively analyze clinical, and radiological findings to avoid the risk of side effects by unnecessary steroid treatment, and we should

Table 2 Comparison of serum parameters between non-relapse and relapse groups at initial episode in univariate analysis

\begin{tabular}{|c|c|c|c|}
\hline $\begin{array}{l}\text { Variable (No. without/ } \\
\text { with relapse) }\end{array}$ & Non-relapse group & Relapse group & $P$ \\
\hline \multicolumn{4}{|l|}{ Serum parameters } \\
\hline $\operatorname{WBC}(23 / 10), \mu \mathrm{L}$ & $9021 \pm 3733$ & $7580 \pm 1975$ & NS \\
\hline LDH (23/10), IU/L & $242 \pm 88$ & $235 \pm 63$ & NS \\
\hline $\operatorname{ALP}(23 / 10), I U / L$ & $285 \pm 105$ & $321 \pm 123$ & NS \\
\hline $\operatorname{CRP}(23 / 10), \mathrm{mg} / \mathrm{dl}$ & $10 \pm 7$ & $8 \pm 7$ & NS \\
\hline $\mathrm{KL}-6(23 / 10), \mathrm{U} / \mathrm{ml}$ & $507 \pm 273$ & $440 \pm 341$ & NS \\
\hline SP-D (23/10), ng/ml & $120 \pm 80$ & $120 \pm 41$ & $\mathrm{~N}$ \\
\hline
\end{tabular}

Values are presented as mean \pm SD. Abbreviations: WBC white blood cell, $L D H$ lactate dehydrogenase, $A L P$ alkaline phosphatase, $C R P C$ reactive protein, $K L-6$ Krebs von den Lungen-6, SP-D surfactant protein, NS not significant. $P$ value was not significant for any variable
Table 3 Comparison of chest CT scan findings between nonrelapse and relapse groups at initial episode in univariate analysis

\begin{tabular}{llll}
\hline & $\begin{array}{l}\text { Non-relapse } \\
\text { group }(n=23)\end{array}$ & $\begin{array}{l}\text { Relapse group } \\
(n=10)\end{array}$ & P \\
\hline $\begin{array}{llll}\text { Pattern of lesion } \\
\text { Consolidation }\end{array}$ & $21(91)$ & $9(90)$ & NS \\
Ground-glass opacity & $19(83)$ & $8(80)$ & NS \\
Nodules & $4(17)$ & $2(20)$ & NS \\
Bilateral & $11(48)$ & $9(90)$ & 0.02 \\
Pleural effusion & $2(9)$ & $0(0)$ & NS \\
Traction bronchiectasis & $4(17)$ & $6(60)$ & 0.02 \\
Location of lesion & & & \\
RUL & $18(78)$ & $6(60)$ & NS \\
RML & $10(43)$ & $4(40)$ & NS \\
RLL & $19(83)$ & $7(70)$ & NS \\
LUL & $12(52)$ & $5(50)$ & NS \\
LLL & $11(48)$ & $6(60)$ & NS
\end{tabular}

Reaction to steroid treatment on chest $C T$

Partial remission $\quad 10(43)$

$$
9 \text { (90) }
$$

Values are presented as No. (\%). Abbreviations: RUL right upper lobe, $R M L$ right middle lobe, $R L L$ right lower lobe, $L U L$ left upper lobe, LLL left lower lobe, NS not significant

decide therapeutic dose and treatment period of steroid for each patient.

The present study has some limitations. First, the period of treatment and the timing of steroid tapering were not unified because of retrospective study. Although we statistically found no difference in the duration of steroid treatment or cumulative dose of prednisone, we do occasionally encounter COP relapse during tapering or after steroid treatment is stopped. In addition to the guidelines of steroid treatment proposed by Epler [3] and Schwarz and King [4], Lazor et al. [12] proposed the following standardized GERM “O"P protocol: $0.75 \mathrm{mg} / \mathrm{kg} /$ day prednisone for 4 weeks, $0.5 \mathrm{mg} / \mathrm{kg} /$ day for 4 weeks, $20 \mathrm{mg} /$ day for 4 weeks, $10 \mathrm{mg} /$ day for 6 weeks, and finally $5 \mathrm{mg} /$ day for 6 weeks. Using GERM“O”P, patients received a significantly lower accumulated corticosteroid dose without higher relapse rate, morbidity, or mortality. It was also reported that treatment of first relapse with high-dose corticosteroids was

Table 4 Comparison of steroid treatment between non-relapse and relapse groups at initial episode in univariate analysis

\begin{tabular}{llll}
\hline Variable & $\begin{array}{l}\text { Non-relapse } \\
\text { group }(n=23)\end{array}$ & $\begin{array}{l}\text { Relapse group } \\
(n=10)\end{array}$ & P \\
\hline $\begin{array}{l}\text { Cumulative dose of } \\
\text { prednisone, mg/kg }\end{array}$ & $79 \pm 47$ & $93 \pm 51$ & NS \\
$\begin{array}{l}\text { Average time of steroid } \\
\text { treatment, days }\end{array}$ & $282 \pm 163$ & $281 \pm 174$ & NS
\end{tabular}

Values are presented as mean \pm SD. $P$ value was not significant for any variable. Abbreviation; NS not significant 
associated with a higher rate of treatment side effects without objective benefit. Although severe adverse events due to steroid treatment were not observed in the present study, we expect that protocol as describes above will be proposed in the future by large prospective clinical trials. Second, we could not evaluate the results of pulmonary function tests such as lung volume and diffusion capacity for carbon monoxide because of the lack of available records for review. Third, the number of registered patients in the present study was small because this was conducted in one hospital. Despite the above limitations, we believe this study has valuable implications for clinical practice.

\section{Conclusion}

We identified several clinical factors that differed significantly in frequency between relapse and non-relapse COP patients. Although there were no significant differences by multivariate Cox regression analysis, we suggest that chest CT scans at initial episode are strong candidate predictive factors for relapse. Identification of such factors could be used to guide preventative treatments, thereby preventing patient distress, avoiding unnecessary steroid treatment, and possibly improving long-term prognosis. We hope that the recurrence factors will be clarified by a long-term study in the future.

\section{Abbreviations}

AFOP: Acute fibrinous organizing pneumonia; ALP: Alkaline phosphatase; COP: Cryptogenic organizing pneumonia; CT: Computerized tomography; KL-6: Krebs von den Lungen-6; LDH: Lactate dehydrogenase; SP-D: Surfactant protein D; WBC: White blood cell; WBC: White blood cell

\section{Acknowledgements}

We are grateful to all the patients who participated in the current study.

\section{Funding}

None

\section{Availability of data and materials}

All data used and generated in this study is available from the authors upon reasonable request.

\section{Authors' contributions}

Dr. ZS designed the study and drafted the manuscript. Dr. YK made substantial contributions to analysis and interpretation of data, and editing of the final manuscript. Dr. TH, MY, KO, TH, AK, KS, KK contributed to data collection, analysis and review of this manuscript. All authors read and approved the final manuscript.

\section{Ethics approval and consent to participate}

The Ethics Committee of the Jikei University Hospital approved the current study (No. 25-330). Informed consent was waived because this was a retrospective study.

\section{Consent for publication}

Not applicable.

\section{Competing interests}

No potential conflicts of interest exist with any companies/oranizations whose products or services may be discussed in this article.

\section{Publisher's Note}

Springer Nature remains neutral with regard to jurisdictional claims in published maps and institutional affiliations.

\section{Author details}

${ }^{1}$ Division of Respiratory Diseases, Department of Internal Medicine, The Jikei University Daisan Hospital, 4-11-1 Izumihoncho, Komae-shi, Tokyo 201-8601, Japan. ${ }^{2}$ Division of Respiratory Diseases, Department of Internal Medicine, Atsugi City Hospital, Kanagawa, Japan. ${ }^{3}$ Division of Respiratory Diseases, Department of Internal Medicine, The Jikei University School of Medicine, Tokyo, Japan

Received: 15 March 2018 Accepted: 10 December 2018 Published online: 09 January 2019

\section{References}

1. Cottin V, Cordier JF. Cryptogenic organizing pneumonia. Semin Respir Crit Care Med. 2012;33(5):462-75.

2. Crestani B, Valeyre D, Roden S, Wallaert B, Dalphin JC, Cordier JF. Bronchiolitis obliterans organizing pneumonia syndrome primed by radiation therapy to the breast. The Groupe d'Etudes et de Recherche sur les maladies Orphelines Pulmonaires (GERM"O"P). Am J Respir Crit Care Med. 1998;158(6):1929-35.

3. Epler GR. Heterogeneity of bronchiolitis obliterans organizing pneumonia. Curr Opin Pulm Med. 1998;4(2):93-7.

4. Schwarz Ml, King TE. Interstitial lung disease. 3rd ed. Hamilton, Ontario: B. C. Decker; 1998. viii, 760

5. Cordier JF. Cryptogenic organising pneumonia. Eur Respir J Physiol. 2006; 28(2):422-46.

6. Drakopanagiotakis F, Paschalaki K, Abu-Hijleh M, Aswad B, Karagianidis N, Kastanakis E, Braman SS, Polychronopoulos V. Cryptogenic and secondary organizing pneumonia: clinical presentation, radiographic findings, treatment response, and prognosis. Chest. 2011;139(4):893-900.

7. Yamamoto M, Ina Y, Kitaichi M, Harasawa M, Tamura M. Clinical features of BOOP in Japan. Chest. 1992;102(1 Suppl):21S-5S.

8. Izumi T, Kitaichi M, Nishimura K, Nagai S. Bronchiolitis obliterans organizing pneumonia. Clinical features and differential diagnosis. Chest. 1992;102(3): 715-9.

9. Watanabe K, Senju S, Wen FQ, Shirakusa T, Maeda F, Yoshida M. Factors related to the relapse of bronchiolitis obliterans organizing pneumonia. Chest. 1998;114(6):1599-606.

10. Alasaly K, Muller N, Ostrow DN, Champion P, FitzGerald JM. Cryptogenic organizing pneumonia. A report of 25 cases and a review of the literature. Medicine. 1995;74(4):201-11.

11. Geddes DM. BOOP and COP. Thorax. 1991:46(8):545-7.

12. Lazor R, Vandevenne A, Pelletier A, Leclerc P, Court-Fortune I, Cordier JF. Cryptogenic organizing pneumonia. Characteristics of relapses in a series of 48 patients. The Groupe d'Etudes et de Recherche sur les Maladles "Orphelines" Pulmonaires (GERM"O"P). Am J Respir Crit Care Med. 2000; 162(2):571-7.

13. Davison AG, Heard BE, WA MA, Turner-Warwick ME. Cryptogenic organizing pneumonitis. Quart J Med. 1983;52(207):382-94.

14. Okada F, Ando Y, Honda K, Tanoue S, Matsumoto S, Mori H. Comparison of pulmonary $\mathrm{CT}$ findings and serum $\mathrm{KL}-6$ levels in patients with cryptogenic organizing pneumonia. Brit J Radiol. 2009;82(975):212-8.

15. Nishino M, Mathai SK, Schoenfeld D, Digumarthy SR, Kradin RL. Clinicopathologic features associated with relapse in cryptogenic organizing pneumonia. Hum Pathol. 2014:45(2):342-51.

16. Austin JH, Muller NL, Friedman PJ, Hansell DM, Naidichi DP, Remy-Jardin M, Webb WR, Zerhouni EA. Glossary of terms for $\subset T$ of the lungs: recommendations of the nomenclature Committee of the Fleischner Society. Radiology. 1996;200(2): $327-31$. 